\title{
Efficient and Unique learning of the Complex Receiver Structure of Galileo E5 AltBOC using an Educational Software in Matlab
}

\author{
Subhan Khan, Aisha Batool \\ Corresponding author: *Subhan.khan041@gmail.com
}

\begin{abstract}
This paper presents an efficient and unique method to learn the complex receiver structure of Galileo E5 AltBOC (Alternative Binary Offset Carrier). The software application is designed in Matlab to present each step involved in the design of software receiver of Galileo E5 signal. This software application contains the fundamental concepts of the Galileo E5 signal in the form of signal acquisition, signal tracking, extraction of the navigation data, power spectral density (PSD) of the AltBOC (15, 10), Fast Fourier Transform (FFT) of the E5a and E5b signal and implementation of the subcarrier used for the AltBOC $(15,10)$. This paper also presents the extraction of navigation data by a novel based approach using the prompt channel of carrier tracking from the code loop discriminator.
\end{abstract}

Keywords-AltBOC; Engineering Education; Galileo E5 signal; Signal acquisition; Signal tracking;

\section{INTRODUCTION}

In recent years, software based solutions have made possible the expansion of various tools to support engineering education. The large impact of complex satellite signals in Research and Development (R\&D) proved to be of great worth in the current era. However, many engineers face the ultimate challenge of learning the basic concepts of Galileo signals. The understanding of Auto-correlation function (ACF) of signal acquisition, generation of sub-carrier for AltBOC (15, 10), signal tracking and extraction of the navigation data are very important topics for the researcher of satellite navigation technology. In terms of engineering education, it is aimed that undergraduate or post-graduate students must be prepared with enough knowledge and understanding before working in the field. Therefore, we present one of the simplest and unique software for learning the basic concepts of the baseband signal processing of the Galileo E5 AltBOC signal.

Satellite system that offers continuous positioning over the globe is known as Global Navigation Satellite System (GNSS) [1]. Galileo is European's GNSS at the development stage that consist of E1, E5 and E6 signals. The most sophisticated Galileo E5 signal is split into of E5a and E5b signals and employs AltBOC modulation that can be used as a wideband signal to offer an accuracy of 20 centimetres even with pseudo-range only. AltBOC is a complex signal composed of four codes multiplexed so as to have a constant envelope. The main lobes of the signal span over $50 \mathrm{MHz}$, which means the signal bandwidth is about thirty times larger than the current Global Positioning System (GPS) signal bandwidth and the signal uses complex modulation [2]. Therefore, due to the intricacy of E5 AltBOC signal it presents extraordinarily challenging for the baseband signal processing.

First stage in the baseband signal processing involves signal acquisition that aims to get rough estimation of the Doppler frequency and code delay of the received signal [3]. Tracking is the second stage in the baseband signal processing of Galileo receiver that aims to refine coarse values obtained from acquisition, and keep track of signal properties that may change over time to demodulate the navigation data from a specific satellite [4]. Navigation data extraction is the third stage in the baseband signal processing section of a Galileo receiver. When the signals are correctly tracked then carrier wave and Pseudo Random Noise (PRN) code can be eliminated from the signal to leave only the navigation bits with a period of $20 \mathrm{~ms}$.

The software application presented in this paper can be used by any institution. However, initial goal is to adopt it at the University of Nottingham, UK campus. The main envisioned outcomes of the developed activities were to do the following:

- Motivate researchers to learn complex receiver structure of Galileo E5 AltBOC.

- Upsurge researchers interest in taking research projects related to Galileo signals.

There has been no document related to the software application based learning of Galileo E5 AltBOC signal. However, several papers have already discussed the baseband signal processing of Galileo E5 signal. For example, in [5], the authors have presented the signal acquisition of E5 Galileo signals in Matlab by using parallel frequency search algorithm. The code is developed on the basis of the free code developed by the GPS software defined radio [6]. However, they were unable to perform the signal tracking. In [7], the authors have presented the basis of baseband signal processing without discussing tracking loop parameters that performs major importance in the development of code and carrier loops. Authors in [8] have proposed an 
innovative and efficient solution for the demodulation of the navigation data. However, they did not analyze the performance of the tracking stages in delay locked loops under hazardous situation.

Novel based approach has been addressed in [9] to perform full band along with wiping off the data bias on both E5a and E5b respectively, to take maximum advantage of power. The introduction of mitigation in the code phase multipath by frequency exploitation is used for E5 signal. Another novel based demodulation approach of Side Band Translator (SBT) is proposed in the [10]. The authors have recovered the data channels by frequency shift of the real signals on the received signal, and obtained replicas from the sub-carrier. Once data is acquired then both the frequency shifts are filtered in a separate way to reduce cross correlations. The interferences of channels were not presented in the above paper. However, the need of interferences with adjacent channels is performed in [11]. Therefore, navigation data is extracted and separately acquired in the two BPSK signals by performing demodulated operation. However, there is no signal acquisition performed by the authors. A complete Galileo E5 AltBOC $(15,10)$ receiver is designed by [12]-[14]. It presents fundamentals of acquisition, tracking and demodulation of the AltBOC $(15,10)$. The demodulation and tracking channels are achieved with hardware prototype of the receiver. However, acquisition algorithm is not efficient and Auto correlation function of the structures has less narrow side lobes.

Many authors have focused on engineering education. For instance, [15]-[21], but as discussed earlier that has been no work related to the learning of Galileo E5 AltBOC for the baseband signal processing of Galileo E5 signal. Our software based receiver will provide complete understanding of the signal acquisition, signal tracking and extraction of navigation data.

The organization of the paper is as follows: Section II explains the Galileo E5 signal structure. Section III presents the baseband signal processing (signal acquisition, tracking and data demodulation) of the Galileo E5 signal. Section IV presents a brief overview of the Matlab based application used for the Galileo E5 signal. Section V presents the GUI based results of the receiver and conclusion is discussed in the section VI.

\section{COMPLEX SIGNAL STRUCTURE OF GALILEO E5 ALTBOC}

The complex receiver structure and implementation of the complex envelope has been provided by the documented presented in [22] as follows:

$$
\begin{aligned}
& s_{E 5}(t)=\frac{1}{2 \sqrt{2}}\left(e_{E 5 a-I}(t)+j e_{E 5 a-Q}(t)\right)\left[s c_{E 5-s}(t)-j s c_{E 5-s}\left(t-T_{s, E 5 / 4}\right)\right]+ \\
& \frac{1}{2 \sqrt{2}}\left(e_{E 5 b-I}(t)+j e_{E 5 b-Q}(t)\right)\left[s c_{E 5-s}(t)+j s c_{E 5-s}\left(t-T_{s, E 5 / 4}\right)\right]+ \\
& \frac{1}{2 \sqrt{2}}\left(\bar{e}_{E 5 a-I}(t)+j \bar{e}_{E 5 a-Q}(t)\right)\left[s c_{E 5-p}(t)-j s c_{E 5-p}\left(t-T_{s, E 5 / 4}\right)\right]+ \\
& \frac{1}{2 \sqrt{2}}\left(\bar{e}_{E 5 b-I}(t)+j \bar{e}_{E 5 b-Q}(t)\right)\left[s c_{E 5-p}(t)+j s c_{E 5-p}\left(t-T_{s, E 5 / 4}\right)\right]
\end{aligned}
$$

The respective dashed signal components, $\bar{e}_{E 5 a-I}, \bar{e}_{E 5 a-Q}, \bar{e}_{E 5 b-I}$ and $\bar{e}_{E 5 b-Q}$ represent product signals according to following:

$$
\begin{gathered}
\bar{e}_{E 5 a-I}=e_{E 5 a-Q} \cdot e_{E 5 b-I} \cdot e_{E 5 b-Q} \bar{e}_{E 5 b-I}=e_{E 5 b-Q} \cdot e_{E 5 a-I} \cdot e_{E 5 a-Q} \\
\bar{e}_{E 5 a-Q}=e_{E 5 a-I} \cdot e_{E 5 b-I} \cdot e_{E 5 b-Q} \\
\bar{e}_{E 5 b-Q}=e_{E 5 b-I} \cdot e_{E 5 a-I} \cdot e_{E 5 a-Q}
\end{gathered}
$$

The $e_{E 5 a-I}, . ., e_{E 5 b-Q}$ are Non return to zero (NRZ) signals which comprise the primary $C_{P, E 5 a-I}, . ., C_{P, E 5 b-Q}$ and secondary $C_{S, E 5 a-I}, . ., C_{S, E 5 b-Q}$ codes and navigation message data $D_{E 5 a-I}$ and $D_{E 5 b-I}$. The chip rate of the primary codes is $f_{C h i p}$.

$$
\begin{aligned}
& e_{E 5 a-I}=C_{P, E 5 a-I} C_{S, E 5 b-I} D_{E 5 a-I}=C_{E 5 a-I} D_{E 5 a-I} \\
& e_{E 5 a-Q}=C_{P, E 5 a-Q} C_{S, E 5 a-Q} \\
& e_{E 5 a-I}=C_{P, E 5 b-I} C_{S, E 5 b-I} D_{E 5 a-I}=C_{E 5 b-I} D_{E 5 a-I} \\
& e_{E 5 a-Q}=C_{P, E 5 b-Q} C_{S, E 5 b-Q}
\end{aligned}
$$

Main and auxiliary sub-carrier waveforms can be represented in the form of $S c_{E 5-S}$ and $S c_{E 5-P}$ respectively. In the above expression $T_{s, E 5}=1 / f_{s, E 5}$ is the sub-carrier period and $f_{S, E 5}$ is the sub-carrier frequency. The combination of Galileo E5 signal provides four different combinations in the form of E5a-I, E5a-Q, E5b-I and E5b-Q components, and the auxiliary component for compensation of the signal envelope. 
Quadrature Phase Shift Keying (QPSK) modulation scheme is used in order to modulate E5a-I and E5a-Q components. Similar procedure is used for the E5b sub-carriers. Navigation data channels are E5a-I and E5b-I, also known as data signals. This is due to the modulations in the message bits. Whereas pilot signals are E5a-Q and E5b-Q that contains only pilot data.

\section{DESIGN OF GALILEO E5 RECEIVER}

Receiver side of Galileo GNSS is very complex structure that provides continuous positioning for the incoming signal. Accuracy and precision for the navigation data must be handled carefully for the upcoming technologies. Therefore, this section details the software receiver structure of the Galileo E5 AltBOC.

Satellite raw data is available from the German company (Fraunhofer-Institut fur Integrierte Schaltungem) that passes through antenna and then front-end block as shown in the Fig. 1. The same data is then passed through acquisition stage to give the rough estimation information about the available code phase and frequency. Information obtained from the acquisition is processed by the carrier and code tracking to give the exact information of the availability of particular navigation data. After forming the entire baseband signal processing the whole information is passed to Matlab based Graphical User Interface (GUI). The navigation data is obtained in the form of position, velocity and time (PVT).

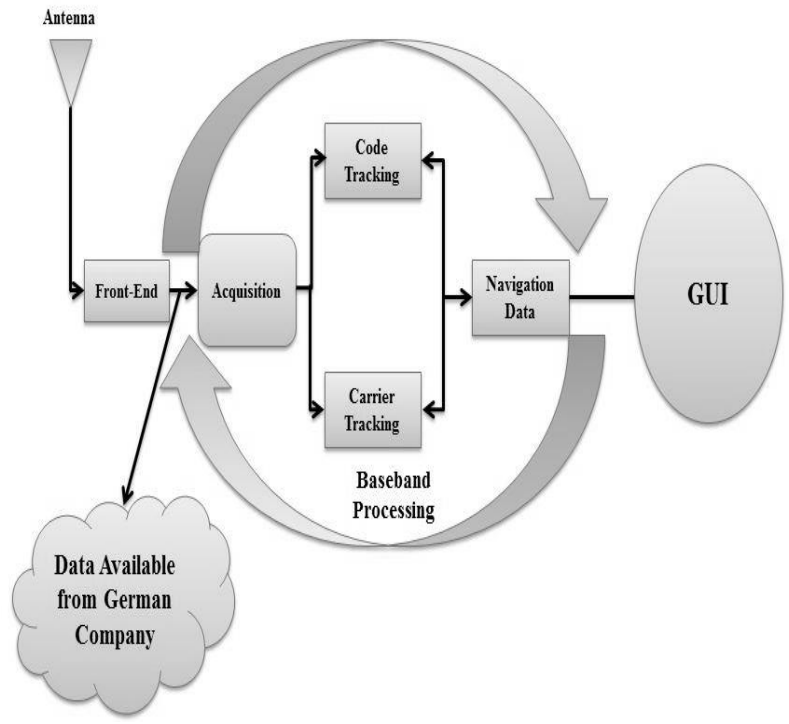

Fig. 1. Receiver design of Galileo E5 AltBOC

\section{A. Signal Acquisition}

Many different approaches have been used for the acquisition process but in this paper, we have used parallel search for the both code phase and frequency search. Parallelism in acquisition can be achieved by either in frequency domain or in the time domain. Analysis of acquisition in time domain is to spread the receiver structure to comprise more correlators, over full spreading code epoch period. While analysis of acquisition in frequency domain is functional in the received stream and locally generated replica code. Since performing correlation in time domain gives a multiplication factor in frequency-domain. Initially the incoming signal is split into In-phase (I) and Quadrature (Q) components. Local oscillator is shifted by 90 degrees to give Q component. FFT is used to combine the both I and Q components and then Correlation is performed by involving the sub carrier of AltBOC and locally generated PRN passed through FFT and complex conjugate. Acquisition ACF is obtained simply by taking Inverse Fast Fourier Transform (IFFT) over the resulting multiplication as shown in Fig 2. The received signal is initially multiplied with a locally produced carrier wave, and giving In-phase (I) and Quadrature (Q) signal components. Both I and Q are shared as a composite input to the FFT. The output of the FFT is then combined with the outcome of the lower branch of the block diagram in Fig 2. 


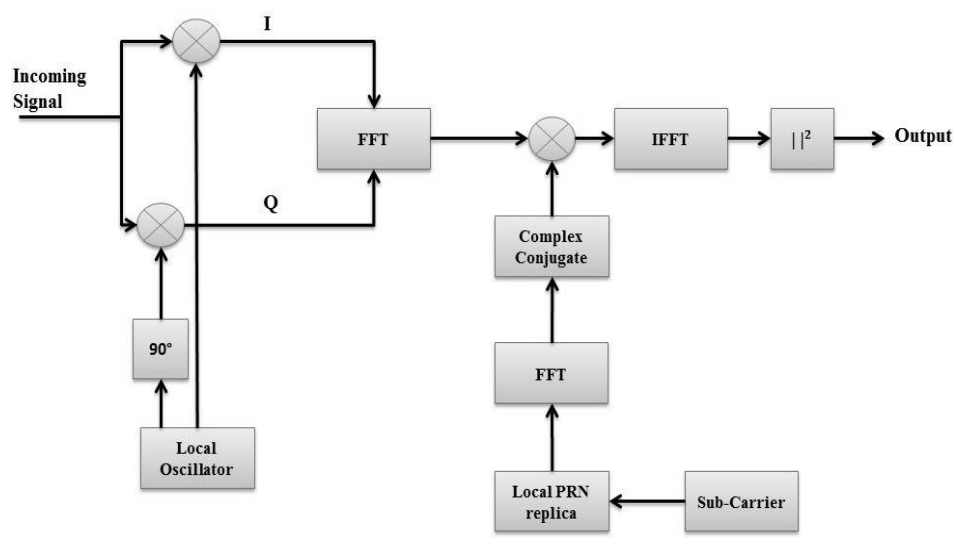

Fig. 2. Parallel code phase search block diagram[24]

Reshape function is used to convert acquisition vector data into the matrix form to acquire 3D plot. Note that the total length of acquisition output is 1000000 samples and a resample matrix of 10230 chips is used for code samples and multiple of 10230 are used to achieve frequency samples. The AltBOC when sampled at $120 \mathrm{MHz}$ will provide perfect side-lobes as mentioned in the literature and can be observed in Fig 3, but at $80 \mathrm{MHz}$ centred lobes will shift upwards due to mismatching of sampling frequency of satellite raw data and AltBOC modulation. Fig 4 also shows the normalised ACF of AltBOC that does match with MATLAB plot shown in [23]:

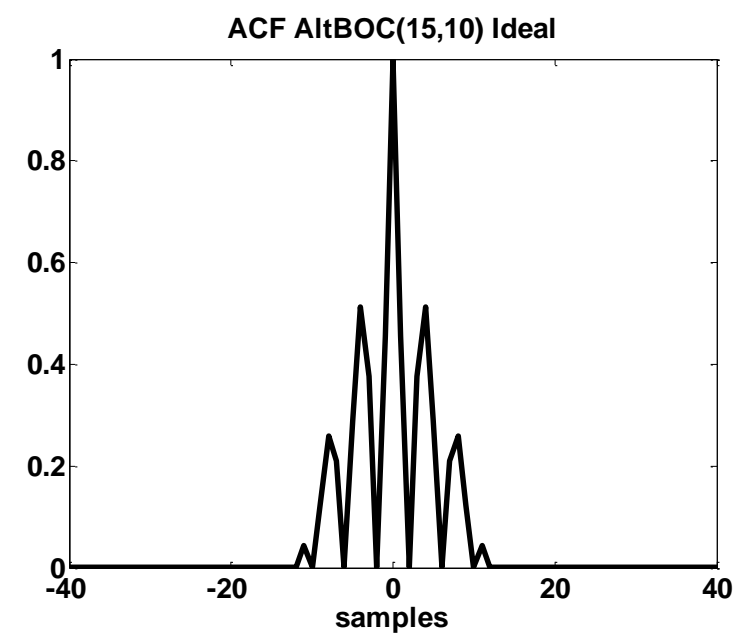

Fig. 3. ACF of AltBOC $(15,10)$ at sampling frequency of $120 \mathrm{MHz}$

\section{B. Signal Tracking and navigation data extraction}

Receiver can lose track of the satellite signal due to the change in the carrier frequency. The carrier tracking loop tries to retain phase lock of the satellite as long as the drift is inside a given boundary. The acquisition will give the carrier frequency within $500 \mathrm{KHz}$, the carrier tracking must then first bring the carrier frequency into lock. We approached the carrier tracking problem by implementing a Phase Lock Loop (PLL). The basic PLL structure is shown in the Fig 4. However, we have made some changes to the architecture. Since we have an integrator that integrates over one PRN code period we get the function of a Low Pass Filter (LPF) with a stop-band frequency of $1 \mathrm{KHz}$. Because of that we leave out the specified low pass filter which is only there to filter out high frequency noise anyway. This approach has been only used for carrier tracking. The intermediate frequency is the carrier frequency fc (this is the frequency that will be corrected) to generate I-component and Q-component. I and Q signals are then multiplied with our prompt replica code and are summed over one period. I and Q signal are then fed into the discriminator which gives us the angle in radians between the I-component and Q-component. What we want is to have the Icomponent as large as possible while keeping the Q-component as small as possible. So, this yields that we should try to keep the discriminator angle as small as possible. The discriminator uses atan instead of atan 2 in order to ignore the phase shifts due to AltBOC. In order to correct carrier frequency, the angle passes from the carrier loop filter to the carrier NCO. 


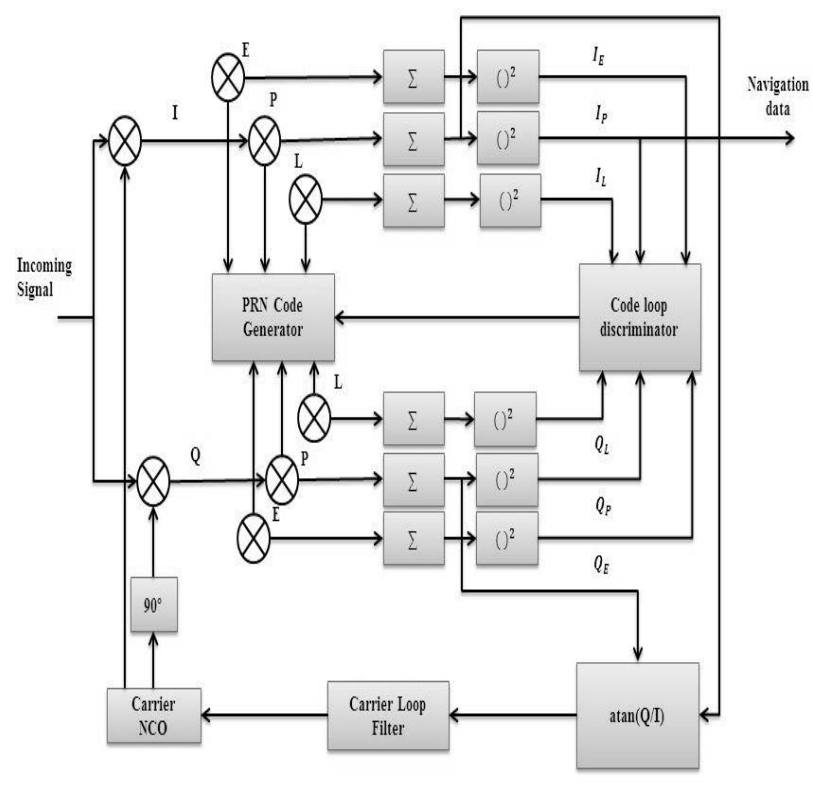

Fig. 4. The block diagram of a complete tracking

\section{channel on Galileo E5 receiver}

Code tracking loop is used to keep track of the code phase of an explicit code in the signal. The result of such a code tracking loop is flawlessly aligned replica of the code. The code tracking loop in the Galileo receiver is a Delay Lock Loop (DLL) called an early-late tracking loop. Correlation ideally slides down linearly from maximum correlation to zero correlation when the phase error is one full chip. If the phase error becomes one full chip, then there should be any correlation since the PRN code is supposed to act like white noise which is uncorrelated. In case of a shift the mark will slide to the right or left, depending on the nature of the shift. Direction of the slide that we have introduced is two additional PRN codes. The new PRN codes are called early, prompt and late PRN. The navigation data is extracted from the prompt code. The discriminator algorithm gives and envisioned or calculated number on the phase error that is present in the receiver. The discriminator output signal $\varepsilon$ is calculated:

$$
\varepsilon=\frac{\text { Early }}{\text { Late }}
$$

The discriminator will give us $\varepsilon=1$, when we are perfectly aligned, $\varepsilon>1$, when we should shift the code to the right, $\varepsilon<1$, when we should shift the code to the left. Since we know that the data provided is from a stationary receiver and have a maximum Doppler shift of approximately $\pm 10 \mathrm{KHz} / \mathrm{s}$, we can calculate the maximum phase deviation per code period as below:

$$
\Delta f_{C A \max }=\Delta f_{c} \cdot \frac{f_{c}}{f_{C A}} \approx 6.5 \text { chips / s }
$$

Where $\Delta f_{C A \max }$ is maximum Doppler shift on carrier, $f_{c}$ is carrier frequency and $f_{C A}$ is the code chipping rate. So to avoid jerky corrections due to noise, we have averaged over twenty code periods, which should be enough to calculate the phase deviation before it reaches a full chip. The worst chip deviation we can obtain per twenty codes is $0.02 * 6.5=0.13$ chips.

\section{BRIEF OVERVIEW OF EDUCATIONAL SOFTWARE}

Educational software for the entire baseband signal processing of Galileo E5 Signal is developed in Matlab using Graphical User Interface Development Environment (GUIDE). The main purpose of developing software application is to arrange data in one place for learning purposes. This standalone application can also be used in the classrooms during the lectures of satellite communication based modules. The first step in the application is to set the value of number of correlators and threshold. A threshold is used to set a point where any particular PRN can be acquired. Number of correlators is used to provide the 
accuracy in the data. However, any large value of correlators will take 10-15 mints to process the entire baseband signal processing.

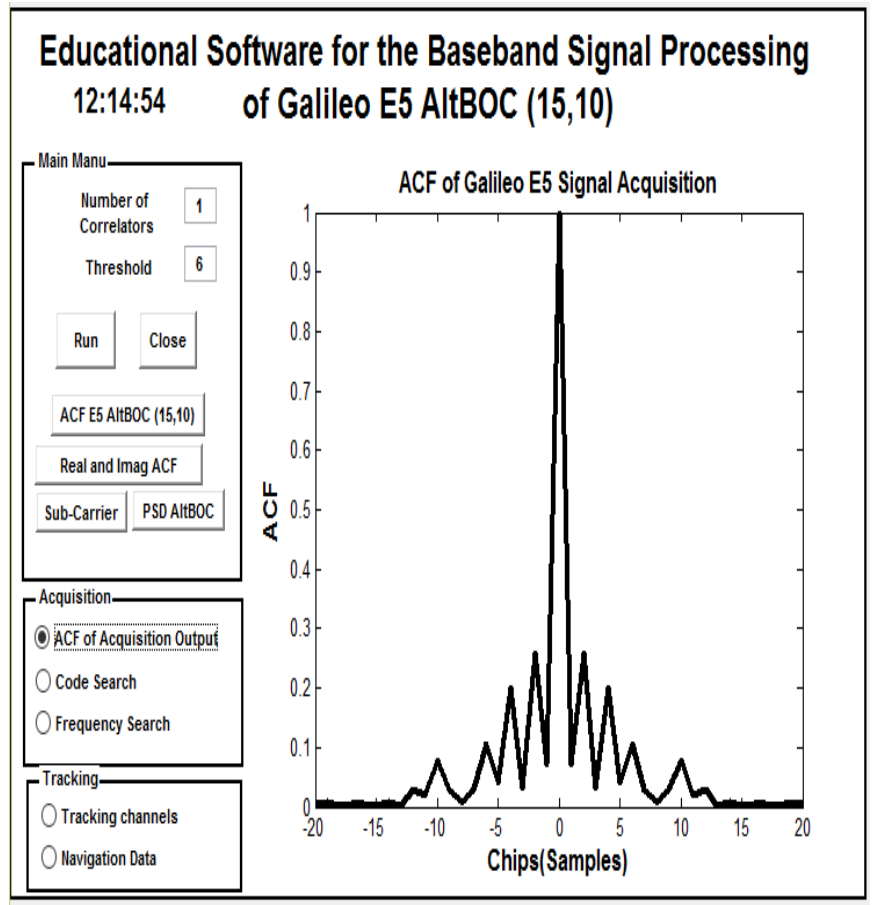

Fig. 5. Initial layout of GUI at $80 \mathrm{MHz}$

Therefore, a range have been set [1-10] for both the threshold and number of correlators between. Initially the values are set 1 and 6 respectively for both number of correlators and threshold.

The educational software works in the following process:

1. Set number of correlators and threshold

2. Press run button to start the acquisition and tracking

In order to view different plots of acquisition and tracking there are separate radio buttons available in the application. Fig 5 shows the ACF of Galileo E5 signal at $80 \mathrm{MHz}$ in the acquisition panel.

For education purposes in the classroom of satellite communications the students can understand the PSD of AltBOC, generation of sub-carrier, working of both frequency and code search, tracking channels in the form of early, prompt and late codes and extraction of navigation data in the form of E5a-I or E5b-I.

\section{RESULTS}

Most conspicuously, ACF of AltBOC is achieved at $80 \mathrm{MHz}$ and higher side peaks of $120 \mathrm{MHz}$ could be compared with Fig. 5. We have sampled both satellite baseband raw data and the AltBOC modulation at the same frequency, there will be higher offset every time due to a mismatch in AltBOC. The only way to obtain perfect result is to remove offset at the time of tracking, which is shown in the Fig. 6 of sub-carrier.

Once the correlator and threshold is set then acquisition can be performed. For the above-mentioned frequency, available from the German company, we have acquired PRN 17. Code search is available for 7.8 MHz. Similarly, for frequency search at 7.8 MHz students can learn the availability of PRN 17. Once the acquisition is successfully performed then tracking can be performed in the GUI. 

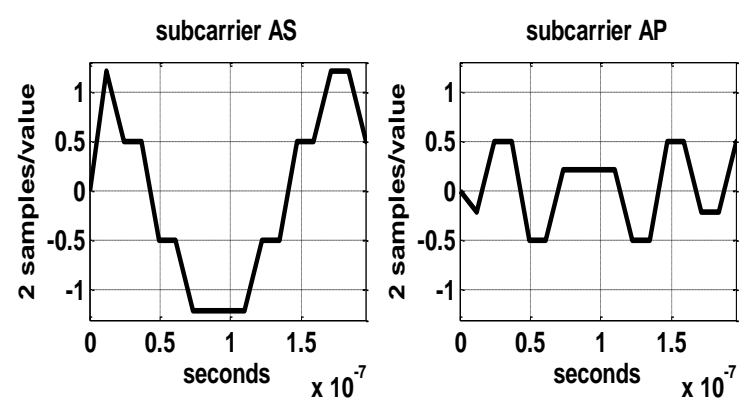

T/4 delayed subcarrier AS
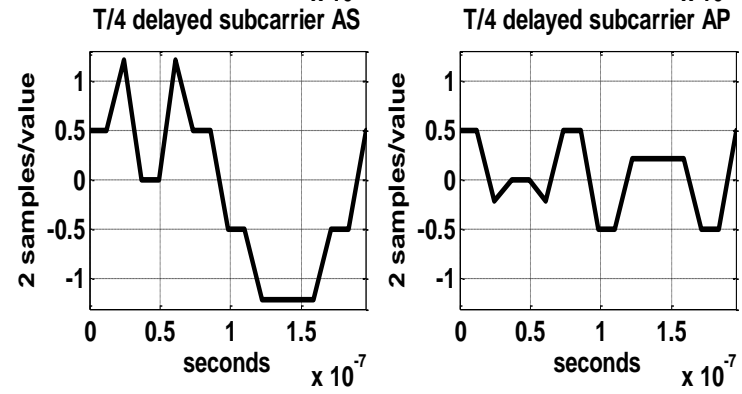

Fig. 6. Generation of the sub-carrier

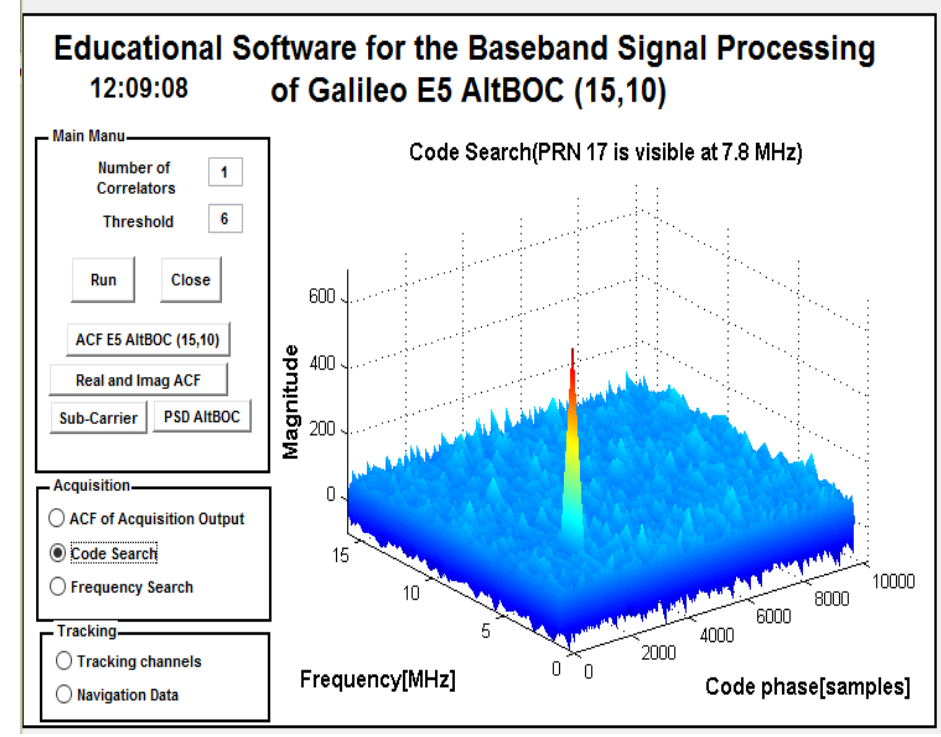

Fig.7. GUI based PRN 17 is Visible in Code Search

The complete acquisition in the form of code phase and frequency search can be observed from the Fig 7 and 8 respectively.

Tracking channels (early, prompt and late) can be seen from the Fig 11. The Matlab application shows that the data channels for late and early gives only delayed and forward replica of them. Whereas the extraction of data channel E5a-I can be retrieved from the prompt channel. Fig 9 shows the navigation data extraction for a complete second. The same can be seen in the ACF form in the GUI. Similarly, E5b-I navigation data can be extracted from the application. The power spectral density of the E5 signal can also be ideally observed from the Fig.10. The only difference between the in-phase and quadrature PSD is that of amplitude spike. 


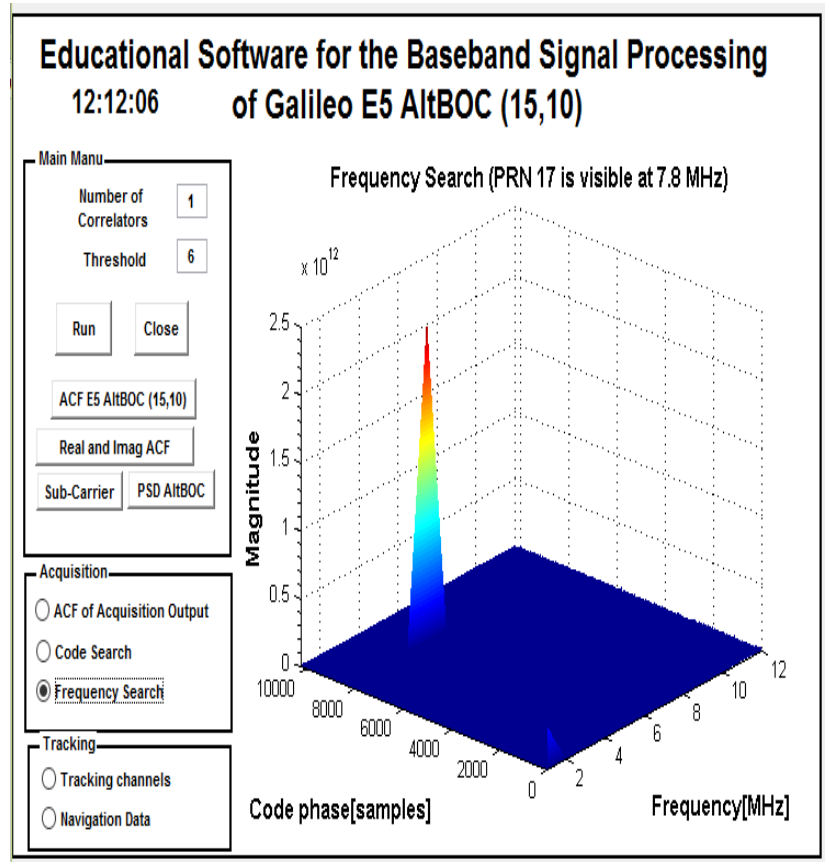

Fig 8. GUI based PRN 17 is Visible in Frequency Search
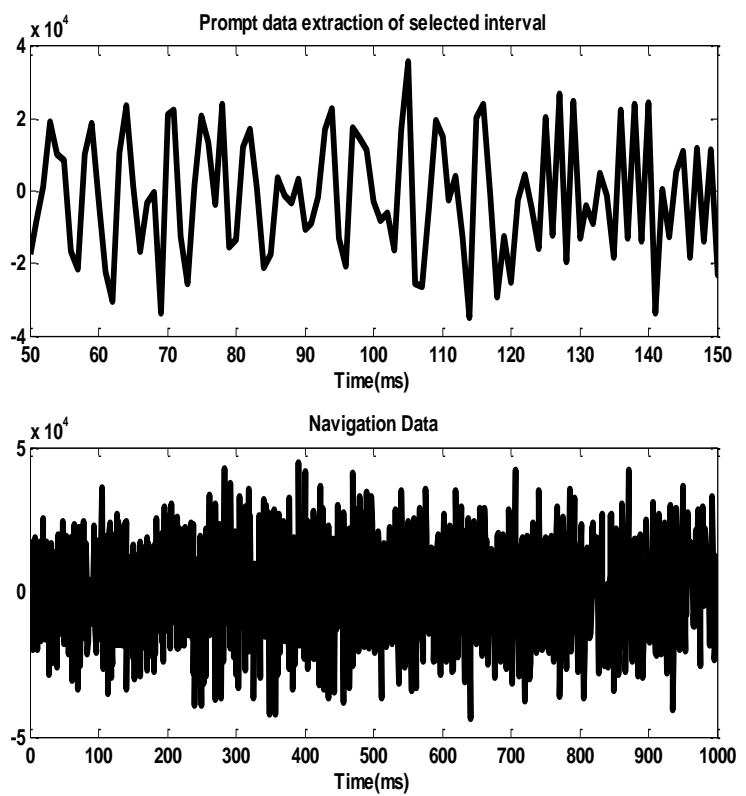

Fig. 9. Navigation data extraction for 1 sec as well as on small selected interval

Students are expected to change the threshold and correlators to observe the change in the behavior of the receiver structure of E5. Phase lock loop works fine with the data provided. The only problem occurs when the acquisition is not close enough to the true carrier frequency. This is overcome by performing a linear fine grained $(\% \mathrm{~Hz})$ frequency search at the fix ed code phase that was obtained from the acquisition process. 

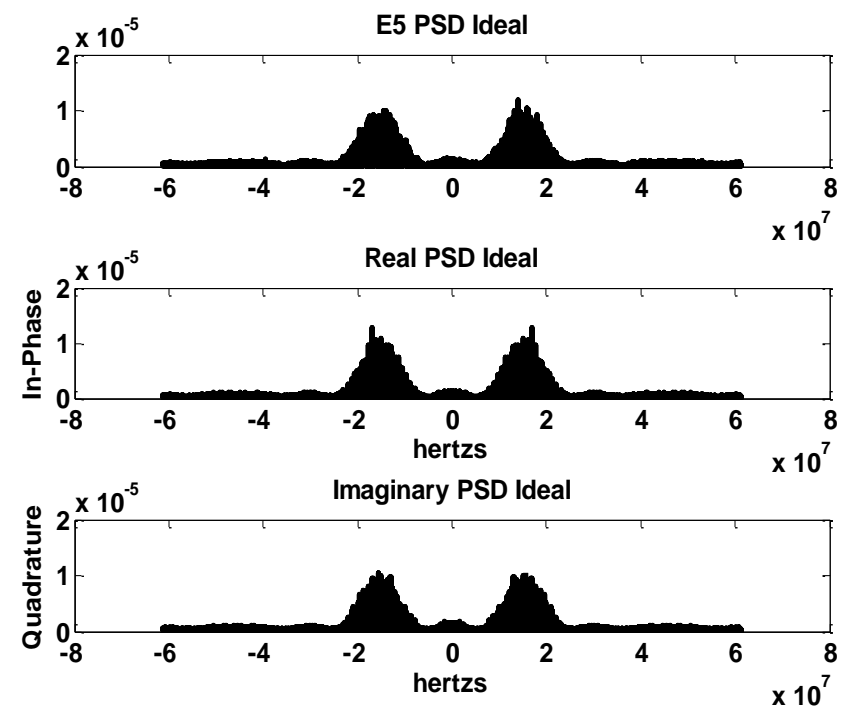

Fig. 10. Power Spectral Density of E5 signal
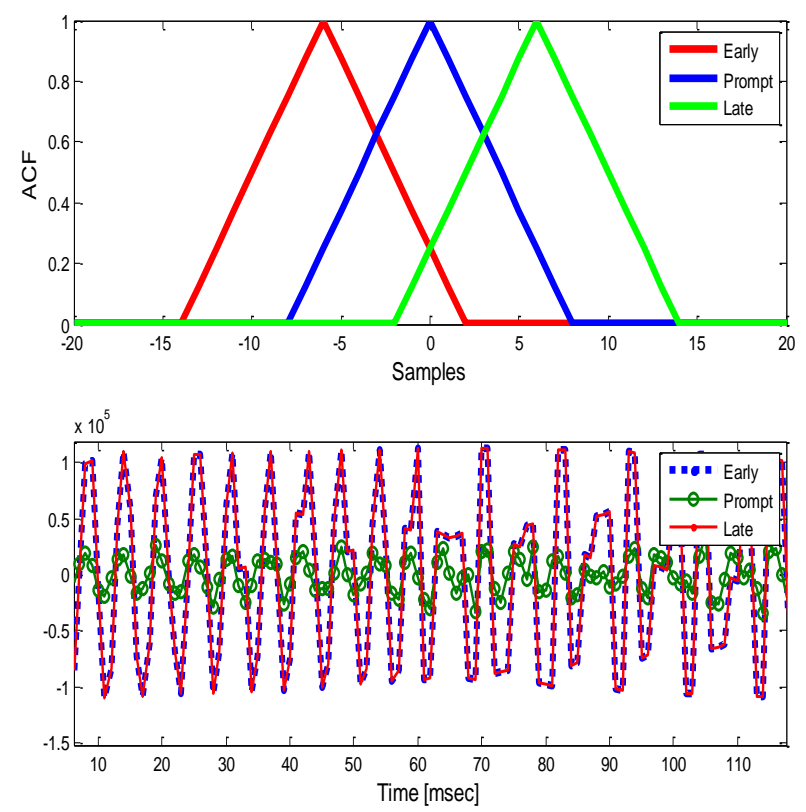

Fig. 11. Tracking channels with ACF of early, prompt and late

\section{CONCLUSION}

A unique method has been proposed for the baseband signal processing of Galileo E5 AltBOC using similar approaches used as in GPS. However, the implementation of code tracking provides efficient novel based technique to keep track of code only by directly sending prompt code in the code loop discriminator. The entire baseband signal processing is performed in one single standalone software application to provide feasibility to researchers and instructors for learning Galileo E5 AltBOC signal. More, importantly, software application provides each step involves in the design of complex receiver structure of Galileo E5 signal during the classrooms. The difference in the frequency provides higher side peaks for the ACF of acquisition but with help of fixed threshold the problem was resolved. 
References

[1] Pajares, H. M., Subirana, J. S., Zornoza, J.J 'Fundamentals and Algorithms', GNSS Data Processing Volume I. : Noordwijk, Netherlands: ESA Communications, 2013.

[2] Jari, N., Elena, S.L., Stephan, S and Heikki, H.: 'Galileo Positioning Technology', Springer, 2015.

[3] Katz, M., "Code Acquisition in Advanced CDMA, Network,"PhD Thesis, University of Oulu, Oulu, Finland, 2002.

[4] Tawk,Y., Botteron,C.,Jovanovic,A., Farine,P.A,:'Analysis of Galileo E5 and E5ab code tracking,' Springer, vol.16, no. 2, pp. 243$258,2012$.

[5] Ekaterina,S.,Ilya,K., Elena,S.L., 'Acquisition of E5 Galileo Signals in Matlab,' Scientific and Technological Experiments on Automatic Space Vehicles and Small Satellites, vol. 104, pp. 36-42, 2015.

[6] Kai,B., Dennis,M.A., Nicolaj,B., Peter,R., Soren, H.J., ‘A Software-Defined GPS and Galileo Receiver,’ Basel: Birkhauser, 2007.

[7] Tawk,Y., Botteron,C., Jovanovic, A., and Farine,P.A.: 'Acquisition performance of Galileo E5a signal,' in Ph.D, Research in Microelectronics and Electronics (PRIME), Berlin, 2010.

[8] Dovis,F., Mulassano,P., Margaria,D.:'Multiresoultion Acquisition Engine Tailored to the Galileo AltBOC Signals,' in ION GNSS, Fort Worth, Tx(USA), 2007.

[9] Shivaramiah,N.C., and Dempster,A.G.: 'The Galileo E5 AltBOC: Understanding the Signal,' in International Global Navigation Satellite Systems Society, Holiday Inn Surfers Paradise, Qld, Australia, 2009.

[10] Margaria,D., Dovis,F., and Mulassano,P.: 'An Innovative Data Demodulation Technique for Galileo AltBOC Receviers,' Journal of Global Positioning System, 2007,vol. 6, no. 1, pp. 89-96.

[11] Ries,L.: 'A software Simulation Tool for GNSS2 BOC Signals Analysis," in Procedings of ION GPS, Portland, Oregon, 2002.

[12] Ries,L: 'New Investigations on Wideband GNSS2 Signals,' in Proceedings of ENC GNSS, Graz, Austria, 2003.

[13] Sleewaegen,J.M.: 'Galileo AltBOC Receiver,' in ION GNSS, Rotterdam, Holland, 2004.

[14] Soellner,M., and Erhard,P.: 'Comparison AWGN Code Tracking Accuracy for Alternative-BOC, Complex-LOC and Complex-BOC Modulations Options in Galileo E5-Band,' in ENC GNSS, Graz, Austria , 2003.

[15] Guzman,J.L.,Costa-Castello,R., Dormido,S., and Berenguel,M.:'An Interactivity-Based Methodology to Support Control Education,' IEEE Control System,2016, vol. 36, no. 1, pp. 63-76.

[16] A. S. ANDREATOS,A.S., and ZAGORIANOS,A.D.: 'ACTA Tools as an Integrated Teaching Assistant for the Design of Automatic Flight Control Systems," in Proceedings of the 6th WSEAS International Conference on ENGINEERING EDUCATION, Heraklion, 2010.

[17] Dash,B., and Vasudevan,V.: 'GUI/Simulink Based Interactive Interface for a DC Motor with PI Controller,' International Journal of Scientific \& Engineering Research, 2011, vol. 2, no. 12.

[18] Su,J.H., Chen,j.j., and Wu,D.S.: 'Learning Feedback Controller Design of Switching,' IEEE TRANSACTIONS ON EDUCATION, 2002,vol. 45, no. 4, pp. 307-315.

[19] Shahnia,F.,Moghbel,M., and Yengejeh,H.H.: 'Motivating Power System Protection Course Students,' IEEE TRANSACTIONS ON EDUCATION, 2016,vol. 59, no. 2, pp. 81-90.

[20] Leo,T., Falsetti, C., Manganello,F., and Pistoia, A.: 'Team teaching for Web enhanced Control Systems education of undergraduate students,' in IEEE EDUCON Conference, Madrid, 14-16 April 2010.

[21] Costas, L.,Fariña,J., and Rodríguez-Andina. 'A configurable framework for the education of digital electronic control systems,' in ELearning in Industrial Electronics, Porto, 3-5 Nov. 2009.

[22] European Space Agency/European GNSS., 'Galileo Open Service. Signal In Space Interface Control Document. OS SIS ICD,' European Space Agency/European GNSS, 2008.

[23] Subedi, P.: 'SOFTWARE SIMULATOR AND SIGNAL ANALYSIS FOR GALILEO E5 BAND SIGNALS,' Faculty of Computing and Electrical Engineering, Tampere University of Technology, Tampere, 2013.

24 Khan, Subhan, Yiqun Zhu, Muhammad Jawad, Muhammad Umair Safder, Mujtaba Hussain Jaffery, and Salman Javid. "Analysis of the satellite navigational data in the Baseband signal processing of Galileo E5 AltBOC signal." arXiv preprint arXiv:2101.01381, 2021 
